\title{
Nonlinear Giant Magnetoresistance in Dual Spin Valves
}

\author{
A. Aziz, ${ }^{1}$ O. P. Wessely, ${ }^{2}$ M. Ali, ${ }^{3}$ D. M. Edwards, ${ }^{2}$ C. H. Marrows, ${ }^{3}$ B. J. Hickey, ${ }^{3}$ and M. G. Blamire ${ }^{1}$ \\ ${ }^{1}$ Department of Materials Science, Cambridge University, Pembroke Street, Cambridge CB2 3QZ, United Kingdom \\ ${ }^{2}$ Department of Mathematics, Imperial College, London SW7 2AZ, United Kingdom \\ ${ }^{3}$ School of Physics and Astronomy, University of Leeds, Leeds LS2 9JT, United Kingdom
}

(Received 1 July 2009; published 4 December 2009)

\begin{abstract}
Giant magnetoresistance (GMR) arises from differential scattering of the majority and minority spin electrons by a ferromagnet (FM) so that the resistance of a heterostructure depends on the relative magnetic orientation of the FM layers within it separated by nonmagnetic spacers. Here, we show that highly nonequilibrium spin accumulation in metallic heterostructures results in a current-dependent nonlinear GMR which is not predicted within the present understanding of GMR. The behavior can be explained by allowing the scattering asymmetries in an ultrathin FM layer to be current dependent.
\end{abstract}

PACS numbers: 75.47.De, 72.25.Ba, 73.43.Qt

The field of spin electronics (spintronics) was initiated by the discovery of giant magnetoresistance (GMR) in magnetic multilayers [1]. GMR arises from differential scattering of the majority and minority spin electrons by a ferromagnet (FM) so that the resistance of a heterostructure depends on the relative magnetic orientation of FM layers separated by nonmagnetic (NM) spacers. GMR is a purely passive phenomenon, but in 1996, Slonczewski [2] and Berger [3] predicted that a large spin-polarized current could exert a spin transfer torque (STT) on a FM layer which has been shown to be sufficient to induce switching between stable magnetization states [4].

The basic theories of GMR [5,6] are linear so that the magnitude of the MR is independent of current density. However, in this Letter, we show that in dual spin valves, magnetic states can be prepared such that the GMR is no longer current independent. We show this currentdependent nonlinear GMR is completely different from STT and is not predicted within the present understanding of GMR. Nonlinear GMR can possibly be explained by allowing the scattering asymmetries in an ultrathin FM layer to be current dependent which must originate from significant changes in the spin-splitting of the density of states. This nonlinear GMR can potentially provide a further active component to the spintronics devices.

Conventional applications of GMR, mainly for reading data from hard discs, use $\mathrm{FM}_{1} / \mathrm{NM} / \mathrm{FM}_{2}$ "spin-valves" (SV) or $\mathrm{FM}_{1} / \mathrm{NM} / \mathrm{FM}_{2} / \mathrm{NM} / \mathrm{FM}_{1}$ dual spin-valves (DSV). In the standard DSV, the $\mathrm{FM}_{2}$ layer switches relative to the parallel-magnetized $\mathrm{FM}_{1}$ layers so that either all three layers are parallel, or adjacent layers are antiparallel; this gives a higher MR than the SV. In our experiments, we measure DSVs which are instead configured so that the $\mathrm{FM}_{1}$ layers are antiparallel; this cancels the conventional linear GMR because switching the $\mathrm{FM}_{2}$ layer retains a configuration such that one pair of FM layers is antiparallel and the other is parallel. This geometry also enables extreme spin-accumulation in ultrathin middle FM layers and so is ideal to probe nonlinear effects; the signature of this is a current-dependent MR which is fundamentally different from STT switching.

In the DSV structures discussed here, the active layers consist of a sputter-deposited $\mathrm{Co}_{90} \mathrm{Fe}_{10}(6 \mathrm{~nm}) / \mathrm{Cu}(4 \mathrm{~nm}) /$ $\mathrm{Py}(x) / \mathrm{Cu}(y) / \mathrm{Co}_{90} \mathrm{Fe}_{10}(6 \mathrm{~nm}) / \mathrm{IrMn}(10 \mathrm{~nm})$ stack [different samples are henceforth labeled as $\operatorname{DSV}(x, y)]$; Py is Permalloy $\left(\mathrm{Ni}_{80} \mathrm{Fe}_{20}\right)$. Thick $\mathrm{Cu}$ layers above and below this stack were used for the bottom and the top electrical contacts, and a 3-D Ga focused ion beam milling technique was used for fabricating current perpendicular to plane nanopillar devices [Fig. 1(a)]; several devices, which behave similarly, were fabricated from each wafer, and fuller fabrication details can be found in previous publications $[7,8]$. The measurements presented here are performed using asymmetric DSVs with $y=2 \mathrm{~nm}$, but symmetrical devices $\operatorname{DSV}(x, 4))$ behave similarly. Single SVs [DSV $(0,2)]$ have also been measured which do not show any current dependence of GMR. All the resistance measurements have been performed at room temperature using a lock-in technique with frequency $77 \mathrm{~Hz}$ and $I_{\mathrm{ac}}=$ $100 \mu \mathrm{A}$. Measurements made at $77 \mathrm{~K}$ are qualitatively similar with small increases in the coercive fields. Positive $I_{\mathrm{dc}}$ corresponds to the electrons flowing from the $\mathrm{IrMn} / \mathrm{CoFe}$ to $\mathrm{CoFe}$ layer.

Figure 1(b) shows the full MR loop of a $\operatorname{DSV}(2,2)$ sample for $I_{\mathrm{dc}}=0$ and $\pm 2 \mathrm{~mA}$. We first concentrate on the MR loop measured at $0 \mathrm{~mA}$ to identify the magnetic configurations corresponding to the various plateaux in the loop. The patterned devices are too small for the magnetic configurations to be measured directly and so careful finite-element magnetic simulations have also been performed to identify the various magnetic configurations (see the supplementary material). At the starting magnetic field $(H)$ of $+150 \mathrm{mT}$, all the magnetic layers are parallel, and so the MR is at its lowest value. When $H$ is decreased to about $50 \mathrm{mT}$, the soft Py layer reverses under the influence of the magnetostatic fields of the parallel-aligned top and 


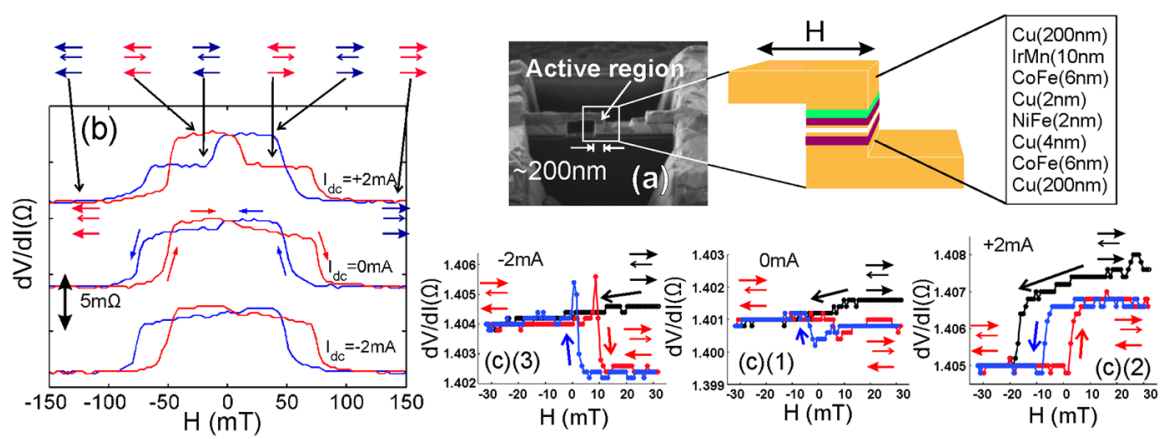

FIG. 1 (color online). (a) Micrograph of a device: the DSV is within the active region where the current flows perpendicular to the plane. (b) Low current full resistance vs field loop of a $120 \times 190 \mathrm{~nm}^{2} \mathrm{DSV}(2,2)$ device; red and blue lines correspond, respectively, to increasing and decreasing field sweeps as indicated by the direction arrows, and the arrows at the top of the graphs represent the magnetic states of the CoFe (top), Py (middle), and CoFe (bottom). (c) Minor MR loops measured at 0 and $\pm 2 \mathrm{~mA}$ : the black curve (uppermost line in each case) is initial sweep from $+150 \mathrm{mT}$ to $-32 \mathrm{mT}$; blue and red curves sweep between $\pm 32 \mathrm{mT}$ with the direction arrows as above.

the bottom CoFe layers. This new state can be viewed as two antiparallel spin valves in series, and so there is a substantial increase in resistance. Further reduction in $H$ results in the reversal of one of the CoFe layers, evident as a small drop in the MR close to zero field, to form a series combination of a parallel and an antiparallel SV. The second CoFe layer reverses at about $-70 \mathrm{mT}$, resulting in a fully parallel state. Although the samples contained an IrMn pinning layer, only a small bias was observable as an asymmetry in the maximal switching fields, but this enables confirmation of the magnetic configurations. The major MR loops at 0 and $-2 \mathrm{~mA}$ are similar, but that at $+2 \mathrm{~mA}$ is substantially modified when the CoFe layers are antiparallel.

To access other states in which the CoFe layers are antiparallel, minor loop measurements were performed in which the system was first saturated at $+150 \mathrm{mT}$ and then loops were measured by sweeping $H$ between $\pm 32 \mathrm{mT}$. Figure 1(c) (1) shows the minor loop for $I_{\mathrm{dc}}=0 \mathrm{~mA}$ : the initial path from $+32 \mathrm{mT}$ to $-32 \mathrm{mT}$ is shown in black, and here the MR decrease is due to the switching of the $\mathrm{CoFe}$ layer as in the major loop. In, contrast, within the stable minor loop, it is the Py layer which reverses close to zero field, and the actual change in resistance is minimal. This is also confirmed from the micromagnetic simulations.

The behavior which is the subject of this Letter is the dramatic change in minor loop shape which occurs when large currents are applied [Figs. 1(c) (2), (3)]. Unlike changes associated with STT-switching, the primary changes are in the magnitude of the resistance corresponding to each magnetic state rather than in the transition fields between them. By symmetry, the field due to the current flowing along the longitudinal sections of the pillar will almost be cancelled at the junction. However, it is likely that the remnant component does result in the small shift in the switching fields seen in the minor loops. The sign of the resistance change on crossing zero field is dependent on the current direction and the magnitude of the change is much larger for $I_{\mathrm{dc}}= \pm 2 \mathrm{~mA}$ than for zero current. A similar current dependence has also been observed for $\operatorname{DSV}(1,2)$ and DSV $(1,4)$ samples and at $77 \mathrm{~K}$.

Figure 2 shows the zero-current major loop (a) and the detailed current-dependence (b) of the stable minor MR loops for $\operatorname{DSV}(1,2)$; those measured at $\pm 1 \mathrm{~mA}$ are strikingly similar to those for $\operatorname{DSV}(2,2)$ at $\pm 2 \mathrm{~mA}$ [Fig. 1(c)]. To compare devices with different Py thicknesses, we plot the field-induced change in resistance-area product $(A \Delta R)$ vs current density for different Py thicknesses [Fig. 2(c)]; the current dependence of $A \Delta R$ decreases rapidly with Py thickness.

In equilibrium (i.e., zero-dc current), the resistance plateaux in the major loops are associated with three distinct magnetic configurations $\uparrow \uparrow \uparrow(P), \uparrow \downarrow \downarrow$ (AP), $\uparrow \downarrow \uparrow\left(P^{*}\right)$; the equilibrium minor loops [Figs. 1(c) (1) and 2(b)] demonstrate that, as expected from the standard Valet-Fert (VF) [5]
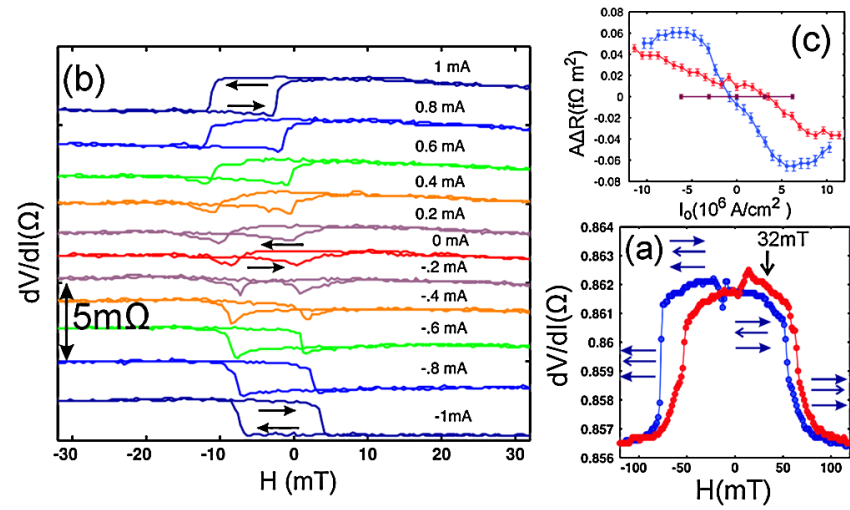

FIG. 2 (color online). (a) Low current full resistance vs field loop of a $\operatorname{DSV}(1,2)$ device; red and blue lines correspond, respectively, to increasing and decreasing field sweeps. (b) A series of minor differential resistance vs magnetic field for a $\operatorname{DSV}(1,2)$ device for dc currents varying from $-1 \mathrm{~mA}$ to $+1 \mathrm{~mA}$. (c) The field-induced change in resistance area product vs current density for $\operatorname{DSV}(1,2)$ (blue), DSV $(2,2)$ (red), and DSV $(8,2)$ (magenta). 
theory, the resistances of the $\uparrow \downarrow \downarrow$ and $\uparrow \uparrow \downarrow$ AP configurations are essentially identical so there is minimal MR.

To reveal the current dependence more explicitly, maps of the minor loop MR for DSV $(1,2)$ are drawn in Figs. 3(a) and 3(b), showing the differential resistance as a function of current and magnetic field for decreasing and increasing $H$, respectively. The diagrams show that, for negative fields, as the current is increased from $-4 \mathrm{~mA}$ to $+4 \mathrm{~mA}$, high resistance states transform gradually (and nonhysteretically) to low resistance states. For positive fields, the opposite it true-i.e., low resistance to high. However, the two maps, and the differences between them, demonstrate that the change in resistance is abrupt (and hysteretic) when the field is swept at fixed current demonstrating that only the field and not the current controls the magnetic state. To confirm this, Figs. 3(c) and 3(d) show that the differential resistance versus current at $0 \mathrm{mT}$ is reversible for both decreasing and increasing field histories, respectively. Since neither curve is hysteretic and they remain distinct proves that current cycling at fixed field does not alter the magnetic state.

Figure 3(e) compares the IR curves of AP and $P^{*}$ states. It shows that $R$ vs $I$ is current dependent only for the AP configurations in which large spin accumulation occurs in the Py: in the $P^{*}$ configuration, the curve is simply the parabolic heating background. The current-dependent MR in the AP configuration therefore has its origin in the asymmetric current dependence of the resistance. The key feature of this current asymmetry is that it is reversible by reversing the magnetic state of the Py layer.
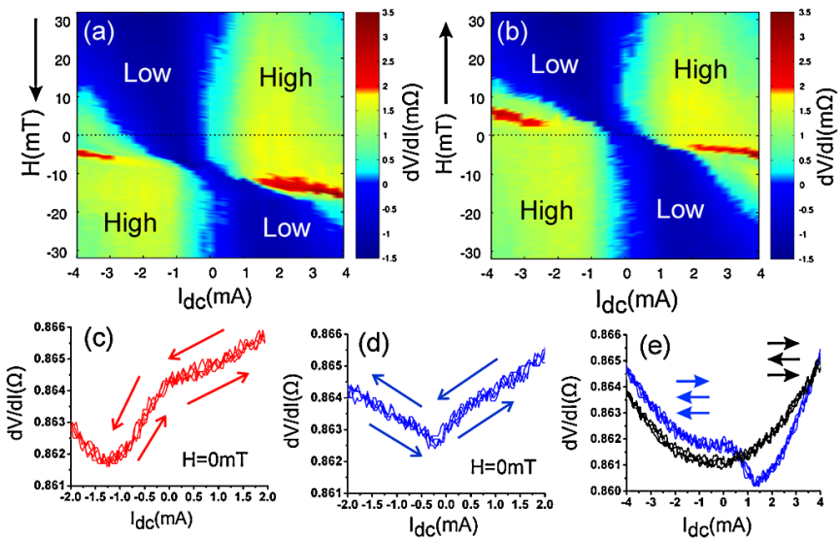

FIG. 3 (color online). Measurements of a DSV(1,2) sample: (a) and (b) MR as a function of dc current for magnet field sweep directions as indicated. Low (dark blue) and high (yellow) resistance states are marked [the peaks (red) are discussed in the text]. In both cases, the parabolic background which is due to Joule heating is subtracted. (c) and (d) are the differential resistance vs $I_{\mathrm{dc}}$ at zero external magnetic fields for the magnetic states of (a) and (b), respectively. (e) Black and blue graphs are the differential resistances vs $I_{\mathrm{dc}}$ at $+20 \mathrm{mT}$ and $-20 \mathrm{mT}$ for the $P^{*}$ and AP states shown using the black and blue arrows, respectively. Joule heating background is not subtracted from graphs (c), (d), and (e).
Figure 4 shows the Py-thickness evolution of the zerocurrent $\mathrm{AP}$ and $P^{*}$ resistances relative to the $P$ saturated state calculated using VF theory. Parameters used in the VF model for calculating the MR are as follows: resistivity of $\mathrm{Cu}, \mathrm{Py} \mathrm{CoFe}$, and $\mathrm{IrMn}$ are 30, 150, 90, and $1000(\mathrm{n} \Omega \mathrm{m})$, respectively. Spin diffusion length $\left(l_{\mathrm{sf}}\right)$ and bulk spin asymmetry constants $(\beta)$ for these materials are $300,4,12$, and $1 \mathrm{~nm}$ and $0,0.13,0.45$, and 0 . Interface resistances for $\mathrm{Cu} / \mathrm{Py}, \mathrm{Cu} / \mathrm{CoFe}, \mathrm{IrMn} / \mathrm{CoFe}$ and $\mathrm{Cu} / \mathrm{IrMn}$ are 0.33, 0.35, 0.95 , and $0.3\left(\mathrm{f} \Omega \mathrm{m}^{2}\right)$, respectively. Corresponding spin asymmetry constants $(\gamma)$ and interfacial spin memory loss parameters are $0.25,0.65,0$, and 0 and $0.25,0.25$, 0.001 , and 0.001 , respectively. While the parameters for other materials are similar to those used elsewhere, values of $\beta_{\text {Py }}$ required to fit the data are substantially smaller than literature values. It is possible that this is associated with Ga ions implantation during fabrication which is known to suppress $T_{C}$ [9] and hence modify the spin asymmetry. It can be seen that there is good agreement with the experimental data. The most notable feature, which is reproduced in our major loop data, is the resistance crossover of the $P^{*}$ and AP states between 1 and $2 \mathrm{~nm}$. This crossover can clearly be seen in the major loops shown in Figs. 2(a) and 1 (a) at $I_{\mathrm{dc}}=0 \mathrm{~mA}$ : in Fig. 2(a) $(x=1 \mathrm{~nm})$, the $P^{*}$ state has a lower resistance than the AP state while the opposite is true for Fig. 1(b) $(x=2 \mathrm{~nm})$.

This zero-current resistance crossover can be understood by considering the limits: for $x=0$, when the device is simply a DSV $(0,1)$ single $\mathrm{SV}$, the AP state reaches its maximum resistance and the $P^{*}$ state is identical to the $P$ saturated resistance. At the opposite extreme, when $x$ is greater than the Py spin diffusion length $l_{\mathrm{sf}}$, the device is effectively two spin valves in series so that $R_{\mathrm{AP}}<R_{P^{*}}$ [as

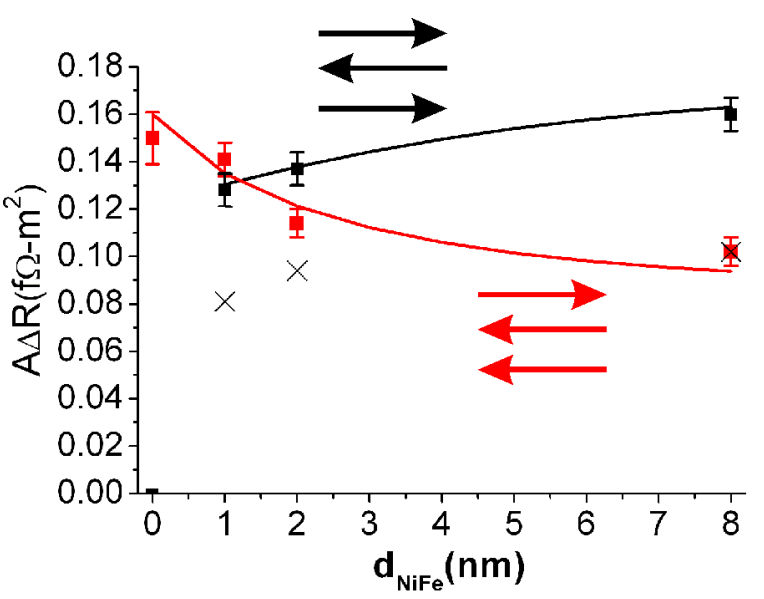

FIG. 4 (color online). Experimental values of the zero-current specific MR of the AP $(\uparrow \downarrow)$ [red (gray) squares] and $P^{*}(\uparrow \downarrow \uparrow)$ (black squares) configurations relative to $P(\uparrow \uparrow \uparrow)$ as function of the thickness of middle Permalloy layer. Continuous lines are the fits calculated using VF model with parameters given in the text. Black crosses are the AP values at $5 \times 10^{6} \mathrm{~A} / \mathrm{cm}^{2}$ obtained by subtracting the minor loop changes from Fig. 2(c) from the zerocurrent AP values. 
measured for the $\operatorname{DSV}(2,8)$ device]. Figure 4 also shows the resistance of the AP state at $5 \times 10^{6} \mathrm{Am}^{-2}$ (black crosses); the displacement of these points from the zerocurrent equivalents [red (gray) squares] highlights the dramatic increase in the current-induced resistance changes as the Py thickness is reduced.

For antiparallel configurations ( $\uparrow \downarrow$ or $\uparrow \uparrow \downarrow$ ), the spin torque acting on both interfaces of $\mathrm{NiFe}$ is additive. However, we argue that the observed current-dependent MR is not due to the spin transfer torque phenomena. Assuming that the STT is responsible for the change in resistance observed in IR curves shown in Figs. 3(c) and 3(e) creates two main difficulties. First, if STT is changing the magnetization direction of NiFe layer, then the change in resistance $(\sim 2 \mathrm{~m} \Omega)$, excluding the Joule heating contribution, cannot be more than the minor MR at $I_{\mathrm{dc}}=0 \mathrm{~mA}(<0.5 \mathrm{~m} \Omega)$-which is obviously not the case. Second, it is clear that the current-dependence of the MR is definitely nonhysteretic with respect to current whereas field-induced MR (and conventional STT) is hysteretic. If we ignore the first criticism for the time being, then the nonhysteretic behavior is only possible if it is assumed that the STT effect is much stronger than the coercive field of NiFe; however, the coercive fields in Fig. 2(b) are clearly significant and, more importantly, independent of current. We therefore conclude that the current-dependent MR is not due to the STT effect. However, reversible peaks, as appear in red regions in Figs. 3(a) and 3(b), can possibly be associated with STT [8]. These peaks appear at higher current density and are very different from the current-dependent MR.

To confirm that heating played no part in the results, we used the temperature dependence of the device resistance to calibrate the heating effect: for $J<5 \times 10^{6} \mathrm{~A} / \mathrm{cm}^{2}$, which is the most important current range for our experiment, the Joule heating is less than $5 \mathrm{~K}$, and therefore the effect on the MR will be very small.

Having excluded the possibility of STT and Joule heating, we turn our attention to other effects which can result in a current-dependent MR. The existence of the currentdependent MR alone indicates physics beyond the standard models of GMR. From the major hysteresis loops [Figs. 1(b) and 2(a)], it is evident that the currentdependent MR only occurs when the outer CoFe layers are antiparallel (AP). This is the configuration which results in significant spin-accumulation in the Py layer, and so we focus on spin-accumulation and its dependence on the Py thickness, as the underlying cause of the effect. Although spin accumulation is an integral component of the VF model, this is implicitly assumed to take place within rectangular bands so that the spin scattering asymmetries which underlie GMR are unaffected by the effective changes in the chemical potentials $(\Delta \mu)$ within the minority and majority bands.

For the MR to be current-dependent, one of the possibilities is that the effective areas of the Fermi surfaces, and hence the scattering asymmetries, must be substantially modified under the extreme nonequilibrium conditions realized in our experiments. Mathematically, the VF model can accommodate this if we allow the interfacial $(\gamma)$ and bulk $(\beta)$ scattering asymmetries to be current dependent. In these diffusive devices, the voltage drop across the heterostructure is less than $1 \mathrm{mV}$, and so the chemical potential shifts are also small. In a rigid band picture of ferromagnetism in Py, this seems at first sight too small to induce large changes in the scattering asymmetry and hence the MR. We can only speculate as to how this coupling occurs, but one intriguing possibility is that large $\Delta \mu$ (at least in comparison with previous spinaccumulation experiments), coupled with the Fermi level lying on a large gradient in the density of states as it does in $\mathrm{Ni}$, can substantially alter the exchange splitting of the density states as in the Stoner model of ferromagnetism [10] so that the effect is effectively magnified. This would account for the features of Fig. 3, in particular, the dependence of the current asymmetry on the direction of the Py magnetization.

In this Letter, we present the first experimental evidence of current-dependent nonlinear MR. It is shown that the nonlinear MR is not due to a STT effect. Nonlinear MR can arise due to the current-induced modification of the intrinsic magnetic properties of $\mathrm{NiFe}$ layer. DSVs have been very useful in detecting these nonlinearities because an antiparallel configuration of a DSV can be thought of as the magnetic analogue of a Wheatstone bridge such that the linear GMR effects are cancelled and the device is then sensitive to the nonlinear GMR. This configuration also enables large spin-accumulations which might be expected to maximize nonlinear effects. Observation of the nonlinear MR has provided new insight into the GMR effect which has a significant technological and fundamental importance. As shown in Fig. 1(b), our DSV also behaves as a weak diode which can be reversed by switching the Py moment and so could, in an optimized system, form the basis of the programmable logic operations.

This work was supported by EPSRC through the Spin@RT consortium.

[1] M. N. Baibich et al., Phys. Rev. Lett. 61, 2472 (1988); G. Binasch et al., Phys. Rev. B 39, 4828 (1989).

[2] J. C. Slonczewski, J. Magn. Magn. Mater. 159, L1 (1996).

[3] L. Berger, Phys. Rev. B 54, 9353 (1996).

[4] E. B. Myers et al., Science 285, 867 (1999).

[5] T. Valet and A. Fert, Phys. Rev. B 48, 7099 (1993).

[6] P. M. Levy and S.F. Zhang, Phys. Rev. Lett. 79, 5110 (1997).

[7] M. C. Wu et al., Nanotechnology 19, 485305 (2008).

[8] M. C. Wu et al., Appl. Phys. Lett. 92, 142501 (2008).

[9] W. M. Kaminsky et al., Appl. Phys. Lett. 78, 1589 (2001).

[10] E. C. Stoner, Rep. Prog. Phys. 11, 43 (1946). 